University of South Carolina

Scholar Commons

$7-1-2003$

\title{
Design of a Multiband Internal Antenna for Third Generation Mobile Phone Handsets
}

\author{
Mohammod Ali \\ University of South Carolina - Columbia, alimo@engr.sc.edu \\ Gerard James Hayes \\ Sony Ericsson Mobile Communications \\ Huan-Sheng Hwang \\ Sony Ericsson Mobile Communications \\ Robert A. Sadler \\ Sony Ericsson Mobile Communications
}

Follow this and additional works at: https://scholarcommons.sc.edu/elct_facpub

Part of the Electrical and Computer Engineering Commons

\footnotetext{
Publication Info

Published in IEEE Transactions on Antennas and Propagation, Volume 51, 2003, pages 1452-1461. http://ieeexplore.ieee.org/xpl/Recentlssue.jsp?punumber=8 (C) 2003 by IEEE
}

This Article is brought to you by the Electrical Engineering, Department of at Scholar Commons. It has been accepted for inclusion in Faculty Publications by an authorized administrator of Scholar Commons. For more information, please contact digres@mailbox.sc.edu. 


\title{
Design of a Multiband Internal Antenna for Third Generation Mobile Phone Handsets
}

\author{
Mohammod Ali, Member, IEEE, Gerard James Hayes, Member, IEEE, Huan-Sheng Hwang, Member, IEEE, and \\ Robert A. Sadler, Member, IEEE
}

\begin{abstract}
A multiband internal antenna is introduced. The antenna consists of a driven meander-line element and two parasitic elements. The design is particularly unique since it supports the third generation mobile phone handsets where multiband operation is greatly desired. The proposed antenna operates effectively in the AMPS 800 (824-894 MHz), GSM 900 (880-960 MHz), and GSM 1900 (1850-1990 MHz) bands within 2.5:1 (voltage standing wave ratio (VSWR). Detail design criteria with respect to geometrical parameter variation are given. Experimental data (VSWR and pattern) obtained from a laboratory prototype are also presented.
\end{abstract}

Index Terms - Antenna, embedded, handsets, internal, multiband, multifunctional, phone, planar inverted-F antenna (PIFA), third-generation, triple band, wideband, wireless.

\section{INTRODUCTION}

W ITH the rapid growth of wireless communications there is a growing demand for mobile phones that are small, attractive, lightweight, and curvy. This has resulted in the proliferation of handsets with antennas that are internal or hidden within the device. An internal antenna makes the handset look much nicer and compact. In addition, since the antenna is inside the phone it is not prone to breakage or damage, which is commonly encountered with the so-called external stub-type antenna. Currently mobile phones with small internal antennas are already in the market.

Designing an internal antenna for a mobile phone is difficult especially when dual or multiband operation is required. Although obtaining dual-frequency resonance is straightforward, satisfying the bandwidth requirement for the respective communication bands is difficult. Further complications arise when the antenna has to operate in close proximity to objects like shielding cans, screws, battery, and various other metallic objects. The planar inverted-F antenna (PIFA) has become the main candidate for the above application since it performs reasonably well compared to other alternatives when operated close to a ground plane [1]-[3].

For conventional PIFAs bandwidth has been found to be largely dependent on the amount of space available for the antenna. This includes the length and width of the antenna and its height from the printed circuit board on which it is

Manuscript received February 8, 2002; revised April 22, 2002.

M. Ali is with the Department of Electrical Engineering, University of South Carolina, Swearingen Building, Columbia, SC 29208 USA (e-mail: alimo@engr.sc.edu).

G. J. Hayes, H.-S. Hwang, and R. A. Sadler are with the Sony Ericsson Mobile Communications, Research Triangle Park, NC 27709 USA.

Digital Object Identifier 10.1109/TAP.2003.812282 mounted. The height being the most critical parameter. The size of the ground plane does not enter into the equation. However, it has been reported in [4] that the size of the ground plane affects the bandwidth, particularly in the lower frequency band $(900 \mathrm{MHz})$. Nevertheless, ground plane size and shape is generally decided upon at the inception of a product and cannot be changed too much. Commercial PIFAs have been designed with $8 \mathrm{~cm}^{3}$ (40 mm by $20 \mathrm{~mm}$ by $10 \mathrm{~mm}$ ) of volume for dual-band operation. Reducing the height from $10 \mathrm{~mm}$ to 6 or $7 \mathrm{~mm}$ is highly desirable. This however affects the antenna bandwidth quite significantly.

If merger of technologies is considered where both advanced mobile phone systems (AMPS) and global system for mobile (GSM) systems are integrated in one phone, triple-band or even quad-band antennas may be needed. For instance, consider a device that operates in the AMPS 800, GSM 900, and TDMA/GSM $1900 \mathrm{MHz}$ bands. This means that the device operates in the 824-894 MHz, 880-960 MHz, and $1850-1990 \mathrm{MHz}$ bands making it triple band from antenna perspective. In the lower band the required bandwidth is $136 \mathrm{MHz}$ or $15.25 \%$ which is almost twice as much of that required for GSM or AMPS alone. This is no doubt very difficult to obtain with a small PIFA.

In this paper, we present a novel triple-band antenna that consists of a driven meander-line element and two parasitic coupled elements. The geometrical configuration, size, and proximity of the driven and parasitic elements help materialize the desired multiband operation. The complete antenna assembly is internal to the handset. The antenna can be tuned to operate either in the 824-894 MHz, 880-960 MHz, and 1850-1990 MHz bands or in the 824-894 MHz, 880-960 MHz, and 1710-1880 MHz bands. The concept described in the paper can also be used to achieve other triple or multiband operations. The size of the antenna is $50 \mathrm{~mm}$ by $10 \mathrm{~mm}$ by $6 \mathrm{~mm}\left(3 \mathrm{~cm}^{3}\right)$ or less. The analysis of the antenna is conducted using a full-wave method of moments (MoM) software package called IE3D [5]. Experimental prototype has also been built and tested. The present analysis of the antenna does not contain the effect of the user's head and hand on the antenna impedance and pattern characteristics. The effect of the operator head and hand on the proposed antenna is planned to be addressed through future publication.

\section{ANTENNA CONFIGURATION}

The geometry of the antenna and its associated printed circuit board (PCB) is shown in Fig. 1. As can be seen from Fig. 1, there are two metal layers. The bottom layer [Fig. 1(a)] consists 

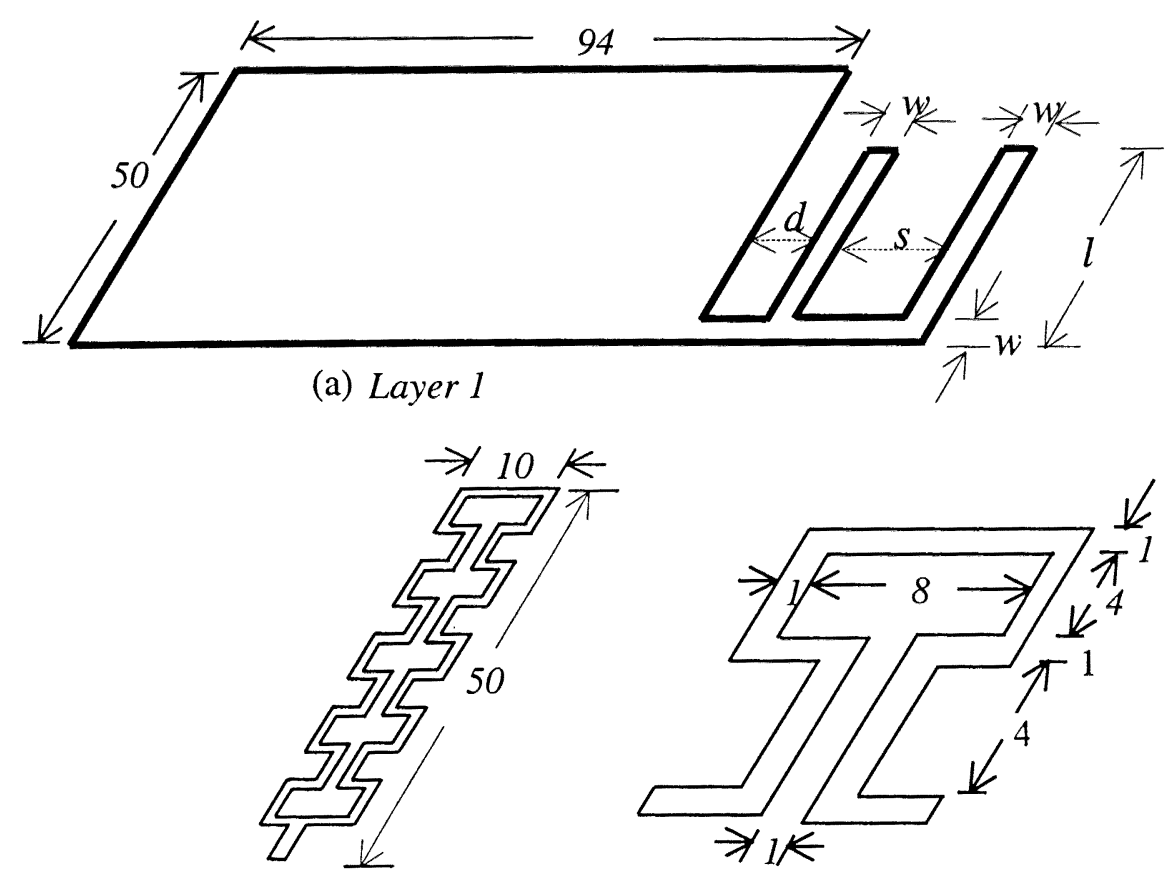

(b) Layer 2

(c) Detail dimension of antenna

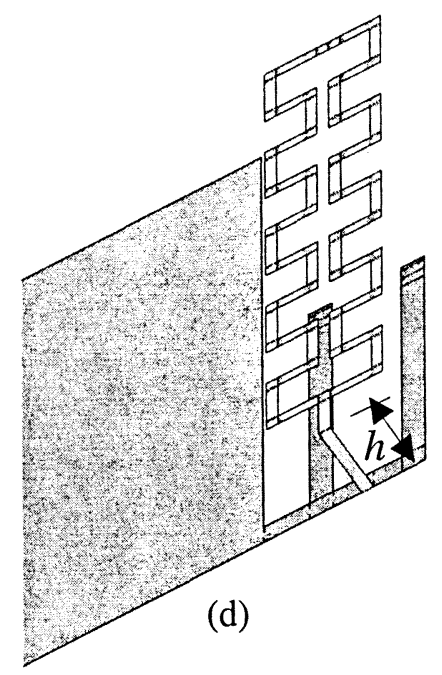

Fig. 1. Antenna and PCB geometry with associated parameters (dimensions in millimeters): (a) PCB and parasitic metal strips, (b) antenna geometry, (c) detail of antenna geometry, (d) antenna height, $h$ from PCB

of a printed circuit board and two parasitic metallic strips. The dimensions of the PCB not including the parasitic elements are $94 \mathrm{~mm}$ by $50 \mathrm{~mm}$. The meander-line element is on the top layer at a height, $h$ from the PCB [Fig. 1(b) and (d)]. We consider air as the dielectric in between the antenna and the PCB. From Fig. 1 the vertical element connecting the antenna and the PCB is the radio frequency (RF) feed. In an actual cellular phone embodiment this will be a signal pin connecting the antenna and the RF signal pad on the PCB.

Other important and relevant parameters of the antenna are also given in Fig. 1 and Table I. The antenna volume is $50 \mathrm{~mm}$ by $10 \mathrm{~mm}$ by $h \mathrm{~mm}$. The distance $d$ is a small separation between one of the parasite and the PCB that can be minimized when
PCB space is critical. The parasites are directly connected to the PCB ground. The antenna is on the upper layer at a height, $h$ from the PCB. The antenna is fed using a connector pin from a RF signal pad on the PCB (not shown).

\section{COMPUTATIONAL Results}

The antenna and the PCB shown in Fig. 1 have been analyzed using IE3D. The double meander geometry for the antenna has been selected for two primary reasons: 1) to shorten the length of the antenna and make it about the same size as the width of the PCB $(50 \mathrm{~mm})$, and 2) to achieve wideband performance [6]. The length of the antenna can be reduced 
TABLE I

List of Antenna And PCB (Printed Circuit Board) PARAmeters and Symbols (SeE Fig. 1)

\begin{tabular}{c|c}
\hline $\begin{array}{c}\text { Fixed antenna and PCB parameters } \\
(\mathbf{m m})\end{array}$ & Variable antenna parameters \\
\hline Antenna length $=50$ & Antenna height $=h$ \\
\hline PCB length $=94$ & Parasitic strip length $=l$ \\
\hline PCB width $=50$ & Distance between nearest parasitic strip and $\mathrm{PCB}=d$ \\
\hline Antenna width $=10$ & $-\cdots$ \\
\hline Width of each parasitic strip $=w=2$ & ---- \\
\hline Spacing between parasitic strip $=s=6$ & ---
\end{tabular}

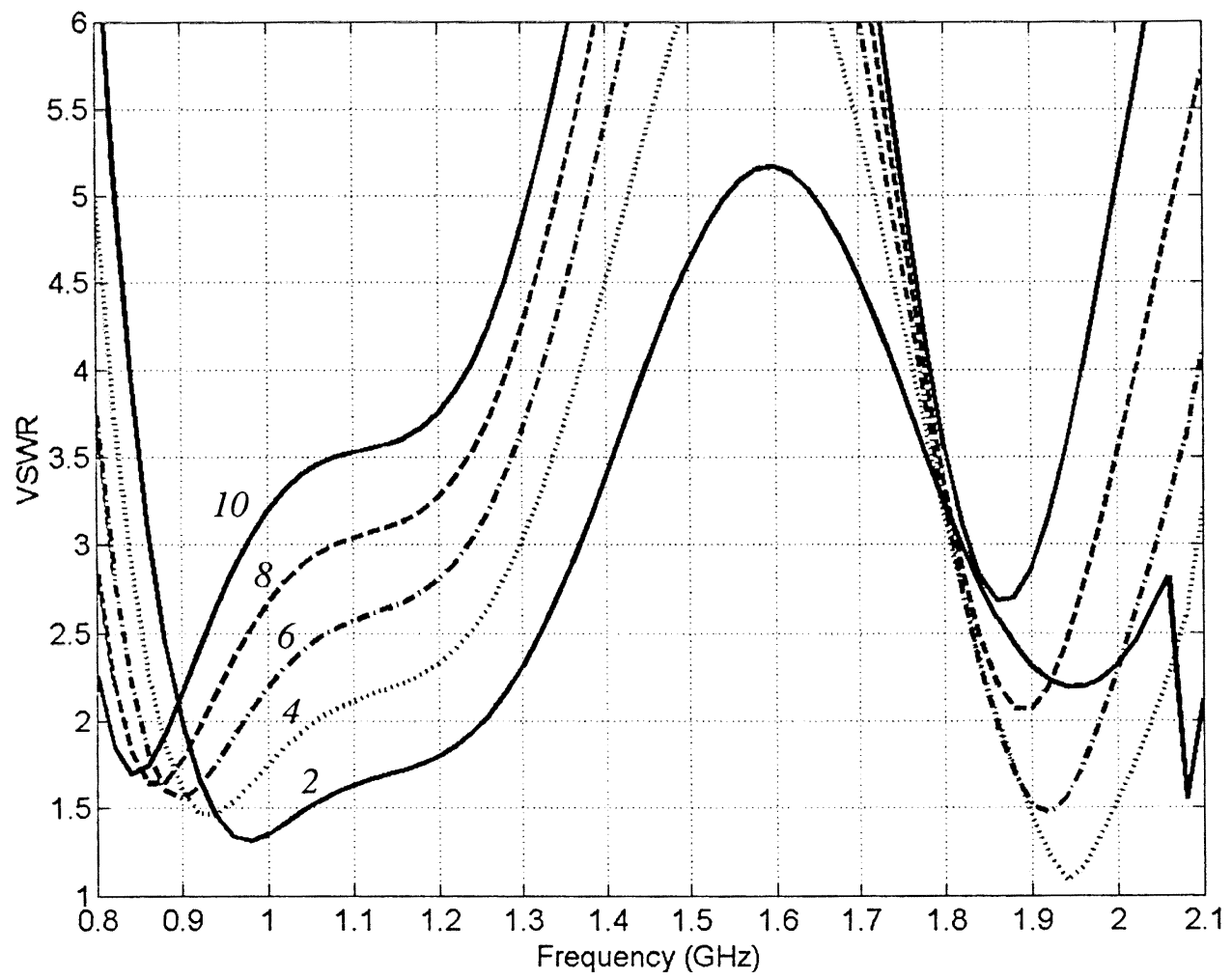

Fig. 2. Computed VSWR versus frequency with antenna height, $h(\mathrm{~mm})$ as parameter, $d=4 \mathrm{~mm}, l=26.5 \mathrm{~mm}$. Other fixed parameters are given in Table $\mathrm{I}$.

even further (current length $=50 \mathrm{~mm}$ ) by increasing its width (antenna width $=10 \mathrm{~mm}$ ). Note that the typical length of a resonant quarter-wave monopole operating around $900 \mathrm{MHz}$ is $80 \mathrm{~mm}$. The double meandering shown in Fig. 1 essentially reduced the antenna length to $50 \mathrm{~mm}$ so that it can be enclosed within the device housing. The other reason for selecting the double meander geometry is its bandwidth advantage over conventional meander-line configuration as has been demonstrated in [6]. The two parasitic metal strips on the PCB help the antenna operate at the DCS or PCS frequency band in addition to its regular operation in the AMPS and GSM bands [6], [7]. The antenna height, $h$ is adjusted to tune the antenna in terms of operating frequency and bandwidth.

Input impedance data for the antenna are computed as function of frequency with antenna height, $h$ as the parameter. VSWR (voltage standing wave ratio) data are computed from the impedance considering a $50 \Omega$ feeding transmission line for the antenna. Computed VSWR data as function of antenna height, $h$ is shown in Fig. 2. The parameters of the antenna are: $l=26.5 \mathrm{~mm}, S=6 \mathrm{~mm}, d=4 \mathrm{~mm}, w=2 \mathrm{~mm}$. Other parameters are given in Fig. 1 and Table I. From Fig. 2 it is apparent that the antenna has two distinct resonances at around $900 \mathrm{MHz}$ and $1920 \mathrm{MHz}$. The first resonance is entirely due to the meander antenna while the second is due to the parasitics that are attached to the PCB [6]-[8]. It is clear that the antenna VSWR changes as $h$ is changed. This change has two aspects: 1) one is the shift in the resonance frequency (as $h$ is reduced the resonant frequencies move higher as expected) and 2) the other is the overall level of the minimum VSWR. The level of VSWR change can be understood from subsequent impedance analysis presented in Figs. 3 and 4.

From Fig. 2, it is also clear that for $h=6 \mathrm{~mm}$, the antenna is very well suited for triple-band operation. In the low frequency band the bandwidth is about $250 \mathrm{MHz}$ or $27.8 \%$ within a VSWR of 2.5:1. This is far larger than the required bandwidth for AMPS 800 and GSM 900 combined (15.25\%). In the high frequency band the antenna operating bandwidth is $9.4 \%$. The required bandwidth for TDMA/GSM 1900 is $1850-1990 \mathrm{MHz}$ or $7.3 \%$. For all practical purposes VSWR of 2.5:1 as an upper limit has been generally found to be acceptable for mobile hand- 


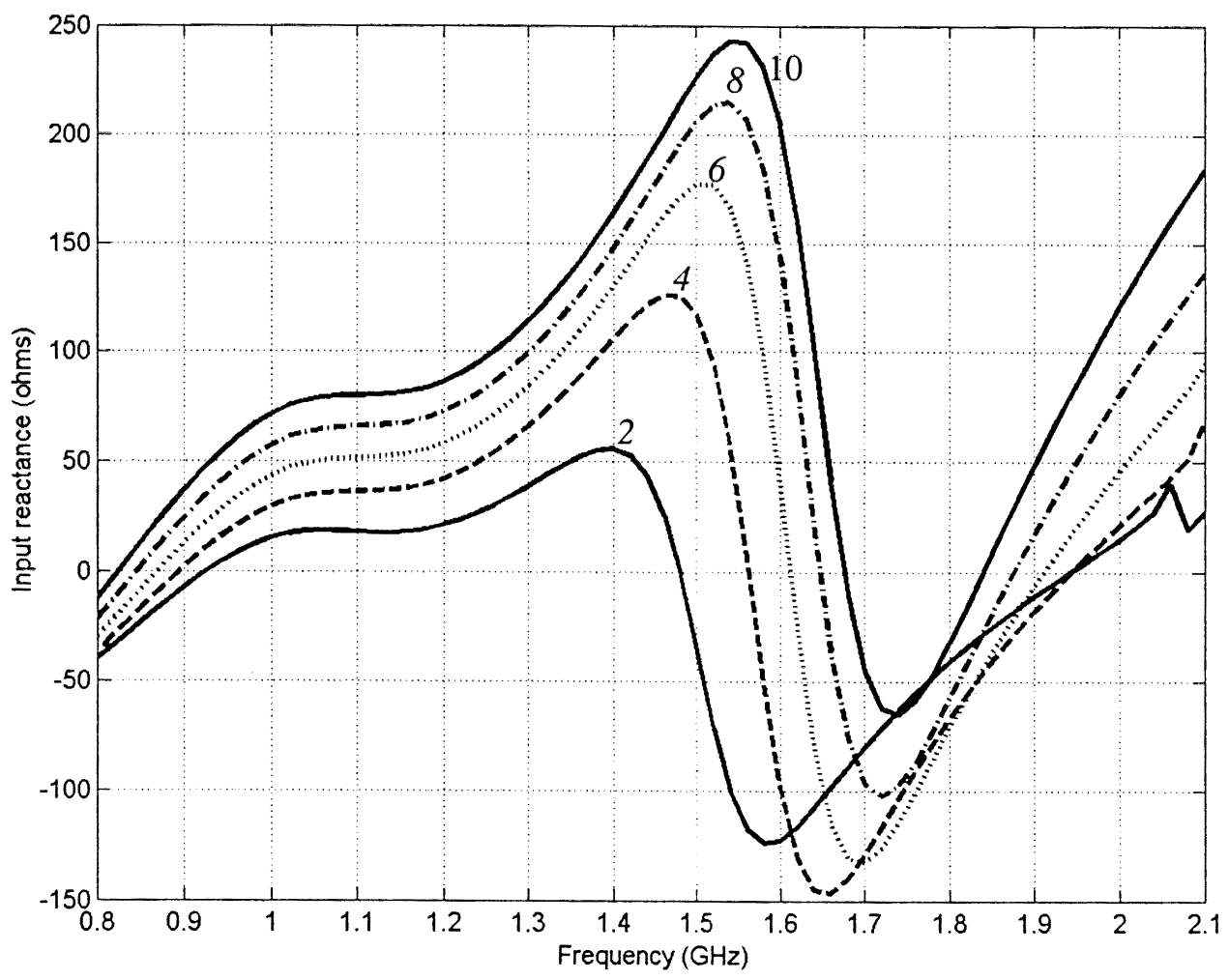

Fig. 3. Computed input reactance versus frequency with antenna height, $h(\mathrm{~mm})$ as parameter, $d=4 \mathrm{~mm}, l=26.5 \mathrm{~mm}$. Other fixed parameters are given in Table I.

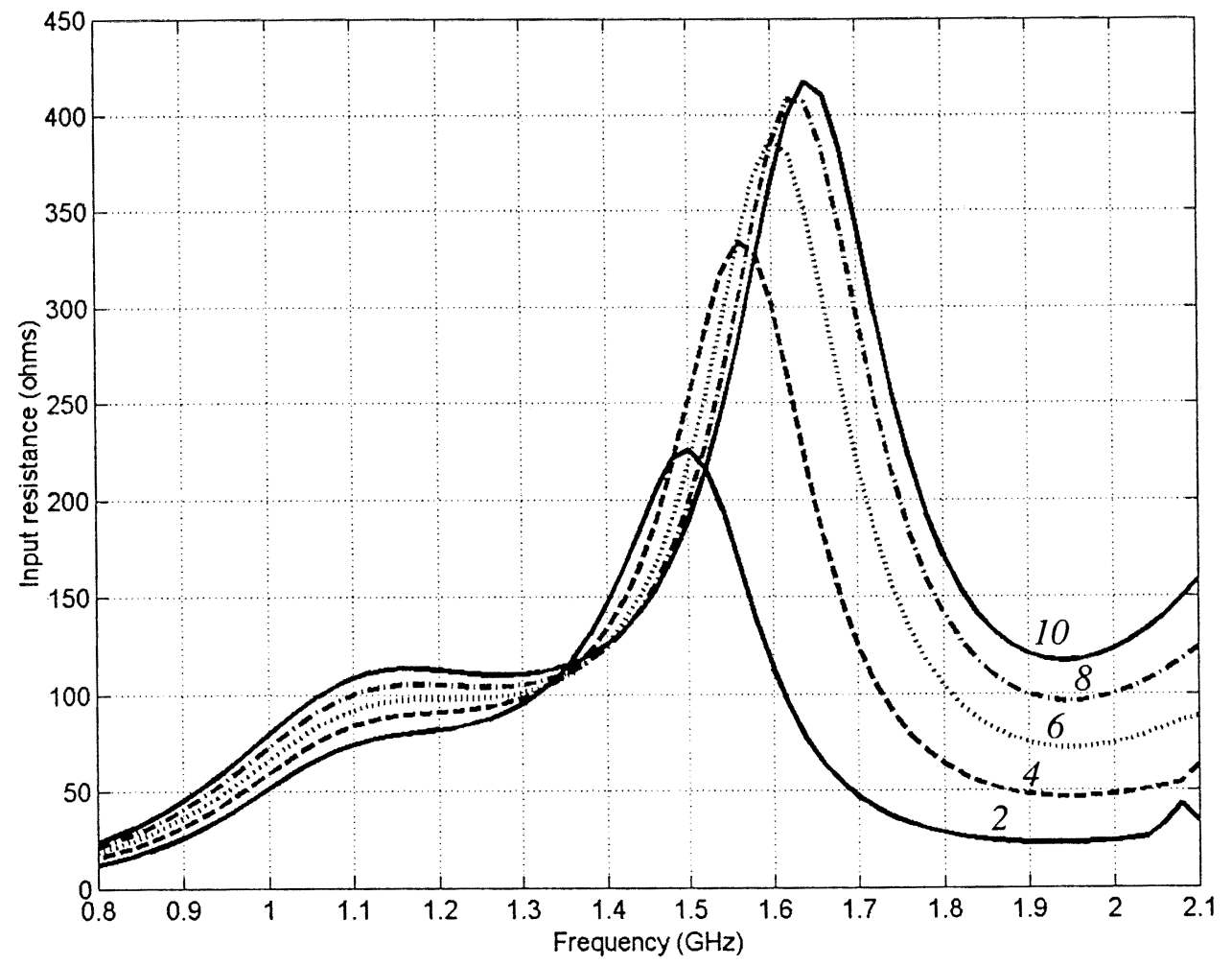

Fig. 4. Computed input resistance versus frequency with antenna height, $h(\mathrm{~mm})$ as parameter, $d=4 \mathrm{~mm}, l=26.5 \mathrm{~mm}$. Other fixed parameters are given in Table I.

sets. There occurs only about an additional $0.4 \mathrm{~dB}$ of loss as the VSWR changes from 2:1 to 2.5:1. The high-band performance is more strongly dependent on $h$ than the low band.
In Figs. 3 and 4 the input reactance and resistance are plotted as functions of frequency with $h$ as parameter. The reactance plot [Fig. 3] clearly shows the change in the resonant 


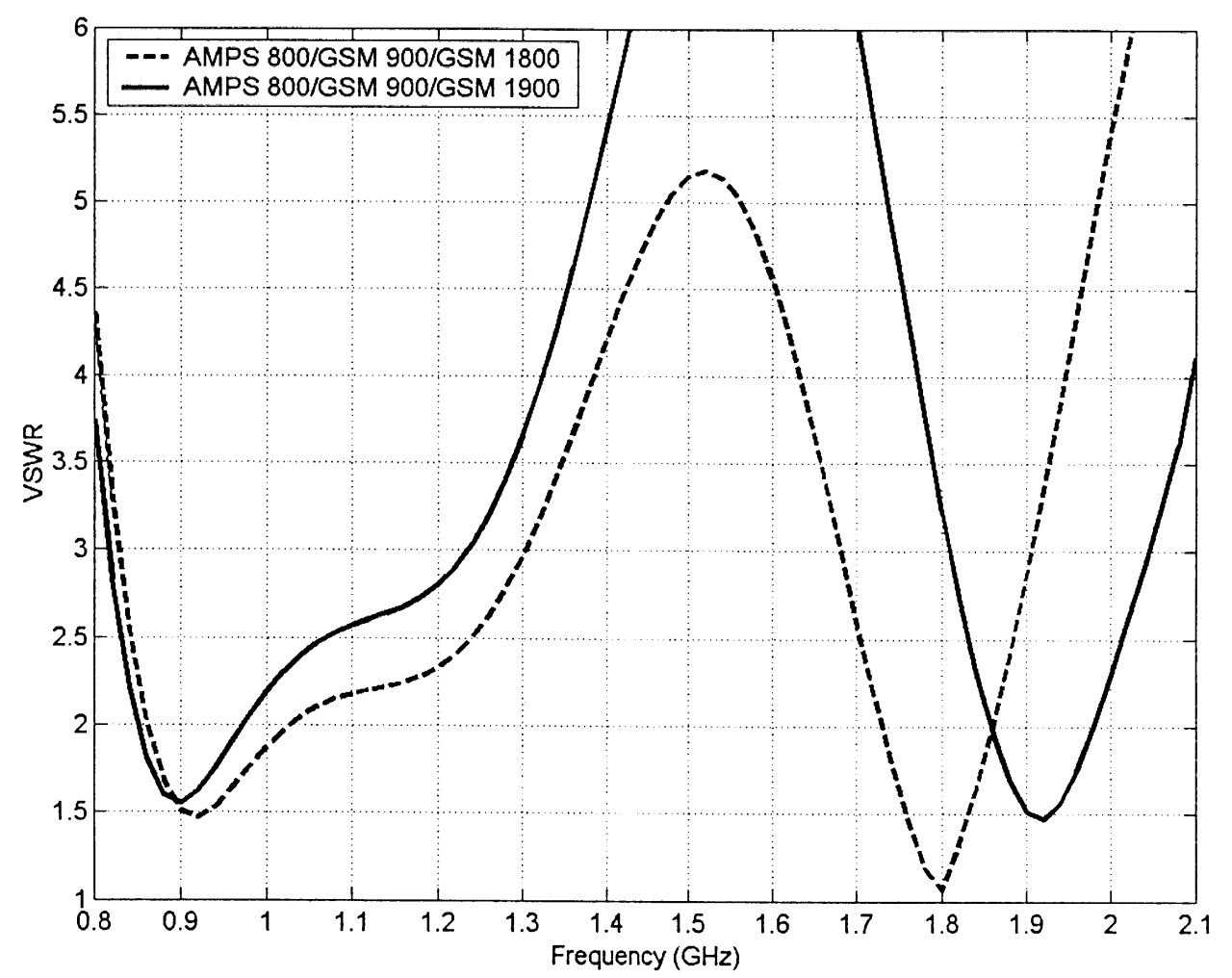

Fig. 5. Computed VSWR versus frequency for AMPS 800/GSM 900/GSM 1800 and AMPS 800/GSM 900/GSM 1900 applications. Solid line- $l=26.5$ mm, $h=6 \mathrm{~mm}$; dotted line- $l=29.5 \mathrm{~mm}, h=5 \mathrm{~mm}$. Other fixed parameters are given in Table I.

frequency as $h$ is changed. The input resistance characteristics of Fig. 4 show that increasing $h$ has much more significant effect in the high frequency band than in the low-frequency band. Clearly in the low band the resistance varies between 25 and $45 \Omega$ at $900 \mathrm{MHz}$ as $h$ changes from 2 to $10 \mathrm{~mm}$. In contrast in the high band the resistance varies between 25 and $125 \Omega$ as $h$ is changed. This is why the VSWR level in the high band depends so strongly on $h$ than in the low band (Fig. 2). Note that $\operatorname{VSWR}=(1+|\Gamma|) /(1-|\Gamma|)$, where $\Gamma=\left(Z_{\text {ant }}-50\right) /\left(Z_{\text {ant }}+50\right)$, with $Z_{\text {ant }}$ being the antenna input impedance consisting of the resistance and reactance of Figs. 4 and 3. The term 50 in the above equation represents the characteristic impedance of a $50 \Omega$ transmission line.

The significant impedance change in the high band can be explained from the coupling mechanism between the driven meander element and the parasitic elements. The coupling is more pronounced in the high band because the low band driven meander element is larger in size compared to the high band parasitic elements. The smaller the height, $h$ the stronger is the coupling. The stronger coupling simply increases the mutual impedance, which in turn reduces the actual driving point impedance in the high band as can be seen from Fig. 4. Thus, when the height is small $(2 \mathrm{~mm})$ the driving point impedance is also small $(25 \Omega)$. In contrast when the height is large $(10 \mathrm{~mm})$ the driving point impedance is also large $(125 \Omega)$. In either of these two cases the match with a $50 \Omega$ feed line will be poor (Fig. 2). Thus, it appears that a height around 4 to $6 \mathrm{~mm}$ can be optimum for the proposed geometry with the parameters defined in Fig. 1.
In Fig. 5, two triple-band operation options are compared. Changing the length of the parasitics, $l$ from $26.5 \mathrm{~mm}$ to $29.5 \mathrm{~mm}$ and the height, $h$ from 6 to $5 \mathrm{~mm}$ the antenna can be used for AMPS 800, GSM 900, and GSM 1800 as opposed to AMPS 800, GSM 900, and GSM 1900 bands. All other parameters are listed in Table I.

In Fig. 6 the effect of the distance $d$ (see Fig. 1 and Table I) on the antenna characteristics is inspected. As $d$ is reduced from 4 to $2 \mathrm{~mm}$ ( $h$ is fixed at $6 \mathrm{~mm}$ ) the resonant frequency in the low band moves higher. This can be taken care of by either physically increasing the length or width of the meander element or by adding dielectric loading in the form of a substrate (support core) or a superstrate (radome). Dielectric loading will essentially reduce the resonant frequency of the antenna since the phase velocity of the wave decreases in dielectric. However, dielectric loading may affect the performance of the antenna in an adverse manner since: 1) the losses in the dielectric can reduce the antenna efficiency, and 2) the dielectric itself may reduce the antenna operating bandwidth. Thus, any dielectric loading has to be closely watched and evaluated so that antenna performance is not degraded. The effect of reducing $d$ from 4 to $2 \mathrm{~mm}$ ( $h$ is fixed at $6 \mathrm{~mm}$ ) in the high band is more significant. It moves the VSWR curve higher $(d=2 \mathrm{~mm}$ and $h=6 \mathrm{~mm}$ as opposed to $d=4 \mathrm{~mm}$ and $h=6 \mathrm{~mm}$ ). This can be solved by reducing the antenna height to $h=5 \mathrm{~mm}$. For $h=5 \mathrm{~mm}$ a very good VSWR response is obtained and the bandwidth requirement for the high band is fulfilled. However, comparing the bandwidth for $d=4 \mathrm{~mm}, h=6 \mathrm{~mm}$, and $d=2 \mathrm{~mm}, h=5 \mathrm{~mm}$, it is evident that the bandwidth in the 


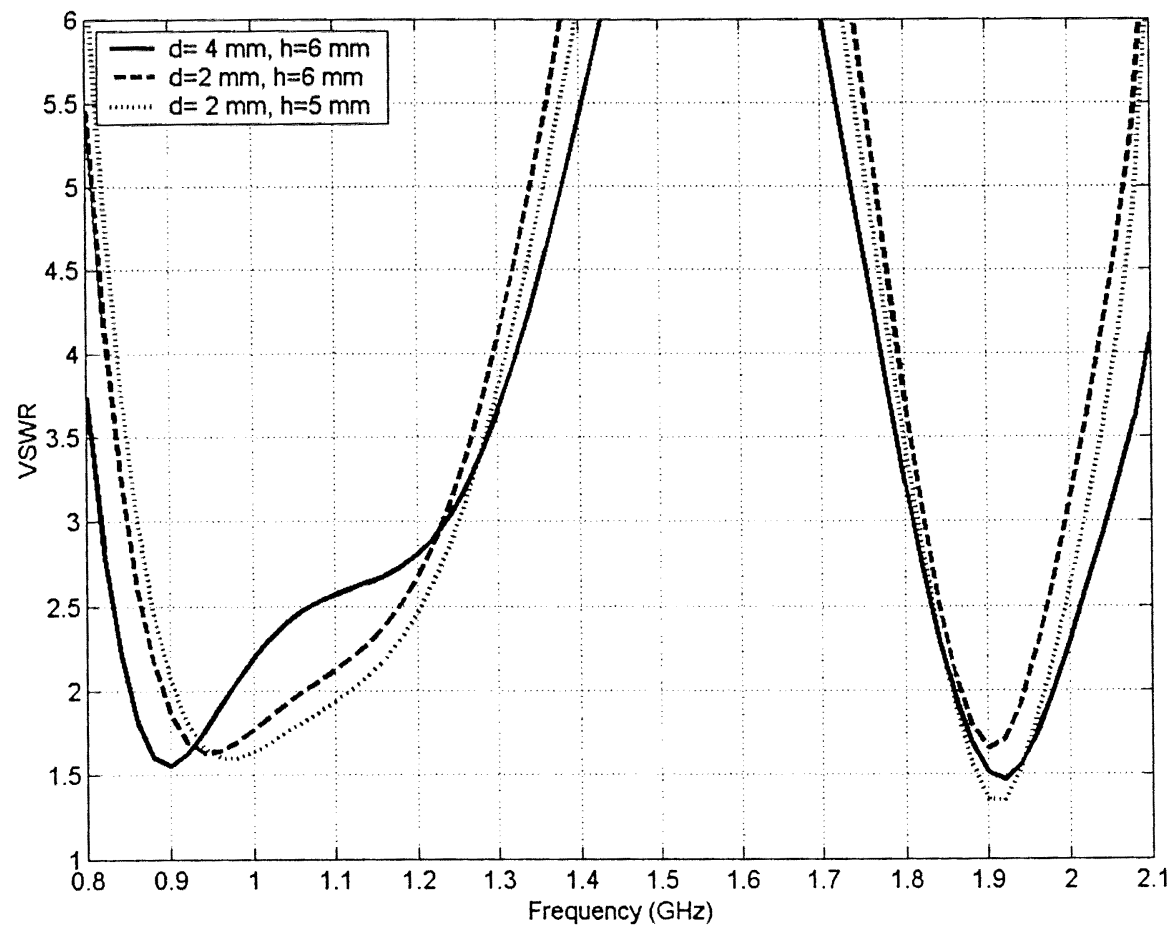

Fig. 6. Computed VSWR versus frequency with $d$ and $h$ as parameters; $l=26.5 \mathrm{~mm}$. Other fixed parameters are given in Table I.

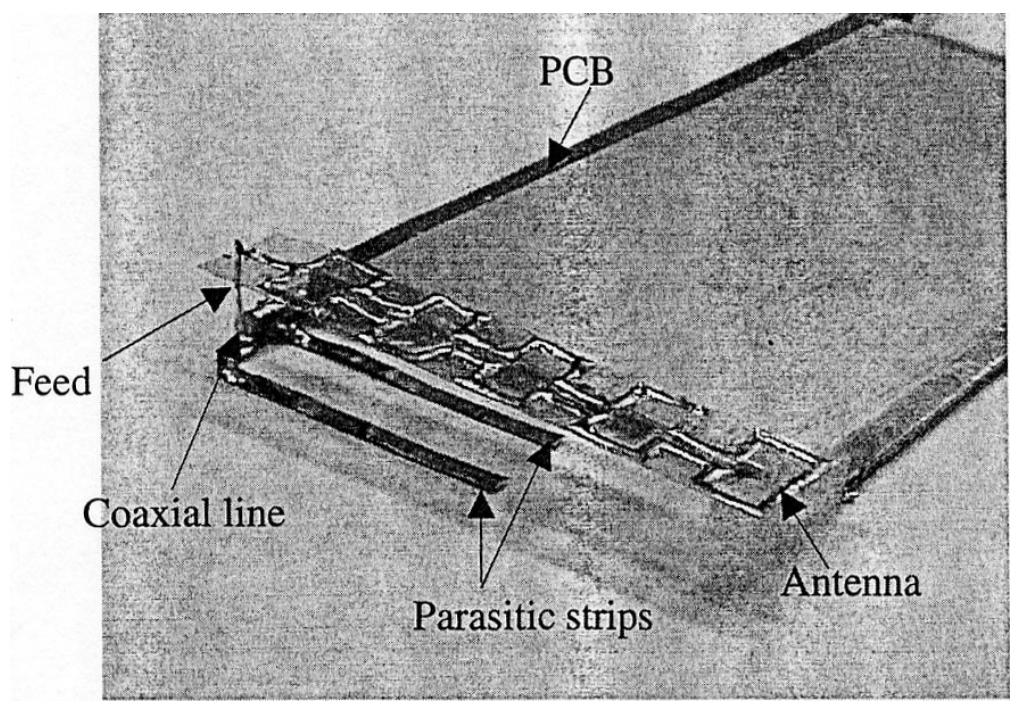

Fig. 7. Laboratory prototype of the proposed antenna. Antenna is placed on a thin transparency film to show all parameters in one picture. In actual measurement a foam $\left(\varepsilon_{r} \approx 1.0\right)$ substrate $6 \mathrm{~mm}$ thick was used to support the antenna.

high band has narrowed as $d$ is reduced. Thus, reducing $d$ even further from $2 \mathrm{~mm}$ will cause the bandwidth to become even narrower.

\section{EXPERIMENTAL RESULTS}

Based on the computational data a prototype antenna was fabricated as shown in Fig. 7 (only the antenna region is shown). A semirigid coaxial transmission line is routed on the FR4 printed circuit board. The center conductor of the coaxial line is connected to the antenna while the shield is soldered to the PCB. For visualization of the antenna, $\mathrm{PCB}$, and parasitic strips the antenna has been placed on a thin layer $(\approx 0.2 \mathrm{~mm})$ of transparency film and then soldered to the inner conductor of the feeding $50 \Omega$ coaxial line. In actual embodiment the antenna lies on a foam substrate $\left(\varepsilon_{r} \approx 1.0\right)$. The substrate measures $50 \mathrm{~mm}$ by $14 \mathrm{~mm}$ by $6 \mathrm{~mm}$ and is sandwiched between the antenna and the parasitic strips. The parasitic metal strips can be seen connected to the PCB ground (Fig. 7). The antenna was measured for VSWR using an HP $8753 \mathrm{E}$ vector network analyzer. Antenna radiation patterns were measured inside an anechoic chamber.

The antenna prototype shown in Fig. 7 resembles the model used for computation (Fig. 1) with $h=6 \mathrm{~mm}$ and $s=6 \mathrm{~mm}$. 


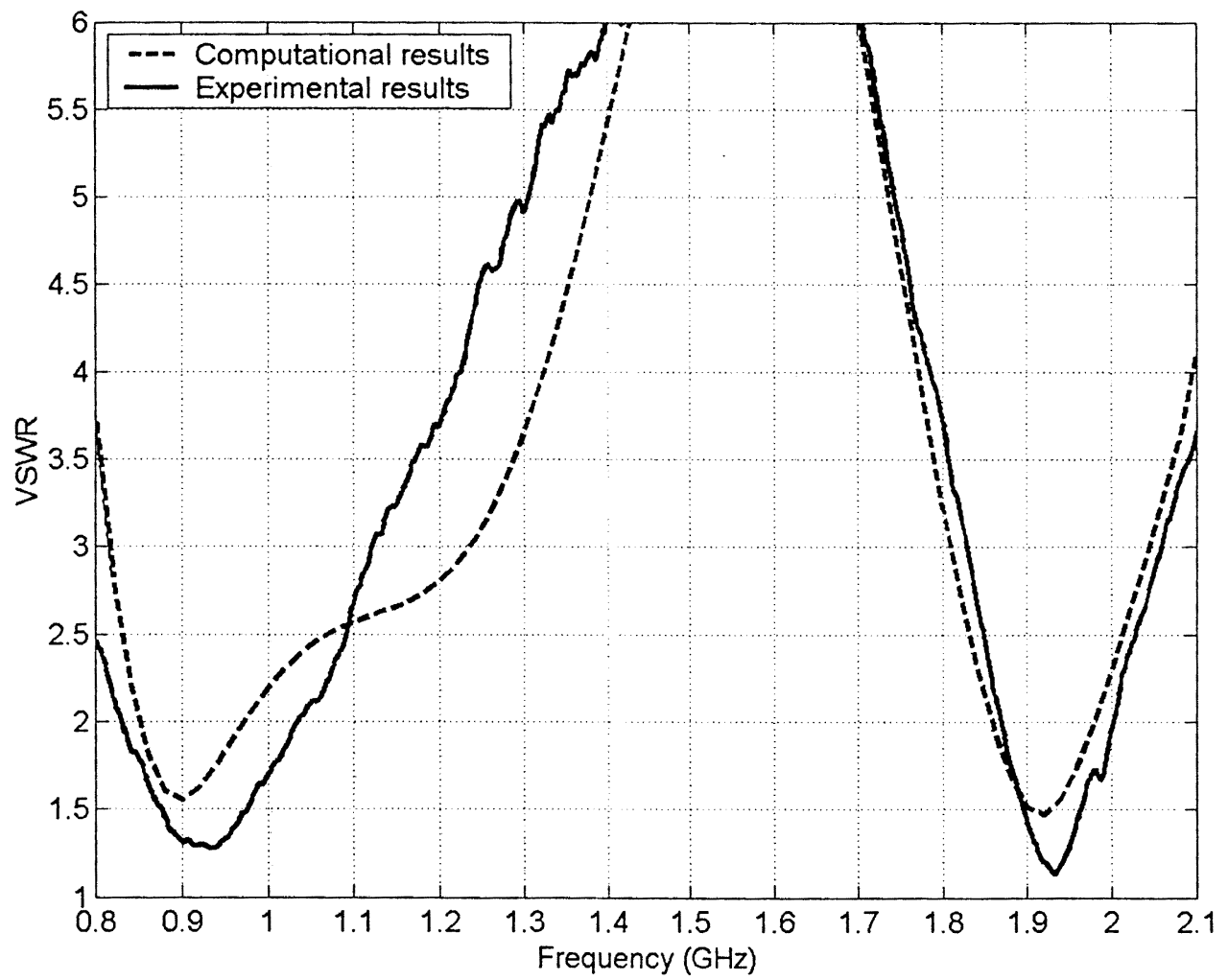

Fig. 8. Computed and measured VSWR versus frequency; $d=4 \mathrm{~mm}, s=6 \mathrm{~mm}$, and $l=26.5 \mathrm{~mm}$. Other fixed parameters are given in Table $\mathrm{I}$.
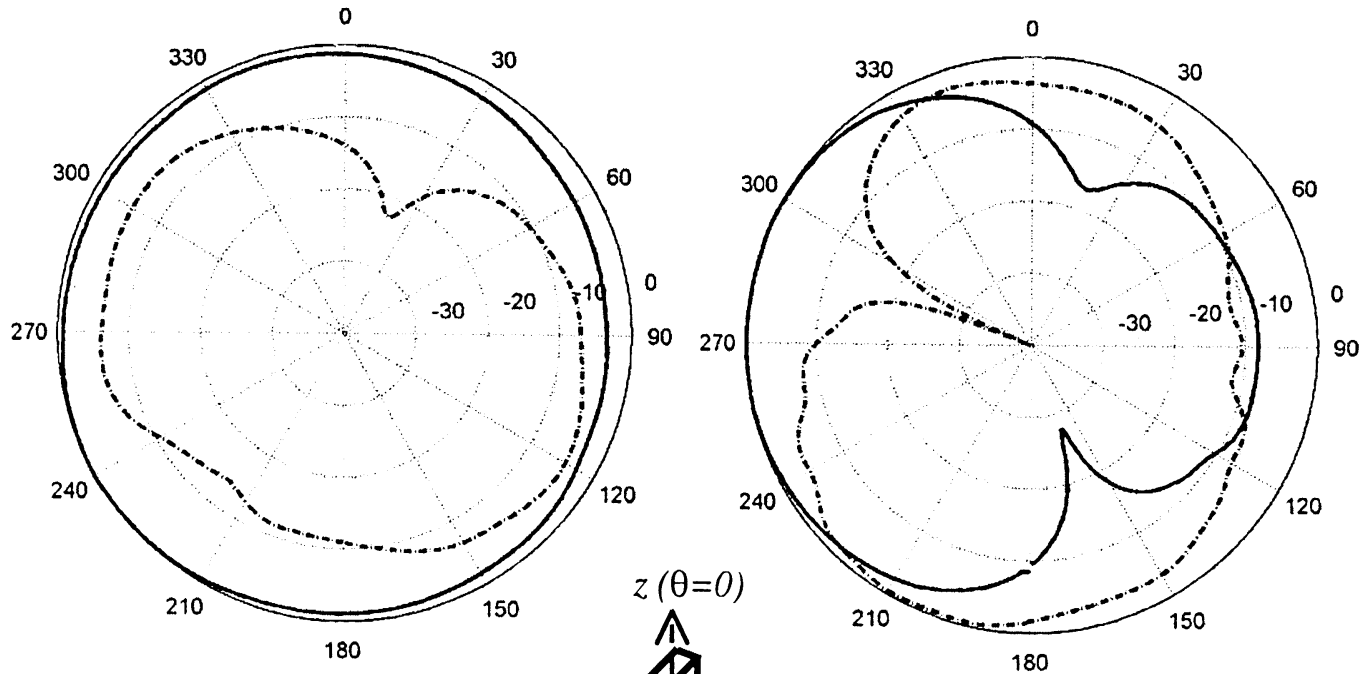

(a)

(a)

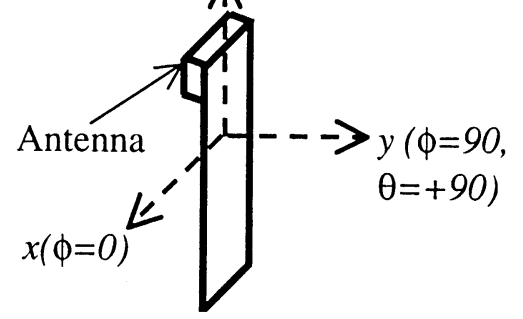

(b)

(b)

Fig. 9. Measured normalized azimuth plane patterns: (a) $x y$ plane (900 MHz), (b) $x y$ plane (1900 MHz). Solid line- vertical component, dashed line-horizontal component; $d=4 \mathrm{~mm}, s=6 \mathrm{~mm}$, and $l=26.5 \mathrm{~mm}$. Other fixed parameters are given in Table $\mathrm{I}$.

Computed and measured VSWR data for this antenna are compared in Fig. 8. The agreement between the measured and the computed data is quite good. Computed and measured resonant frequencies are about the same. The measured bandwidths in both bands are also in good agreement with the computed bandwidths within 2.5:1 VSWR. It is apparent from Fig. 8 that the antenna operates in the AMPS 800 and GSM 900 band within 2:1 VSWR and the GSM 1900 band within VSWR of 2.3:1. 

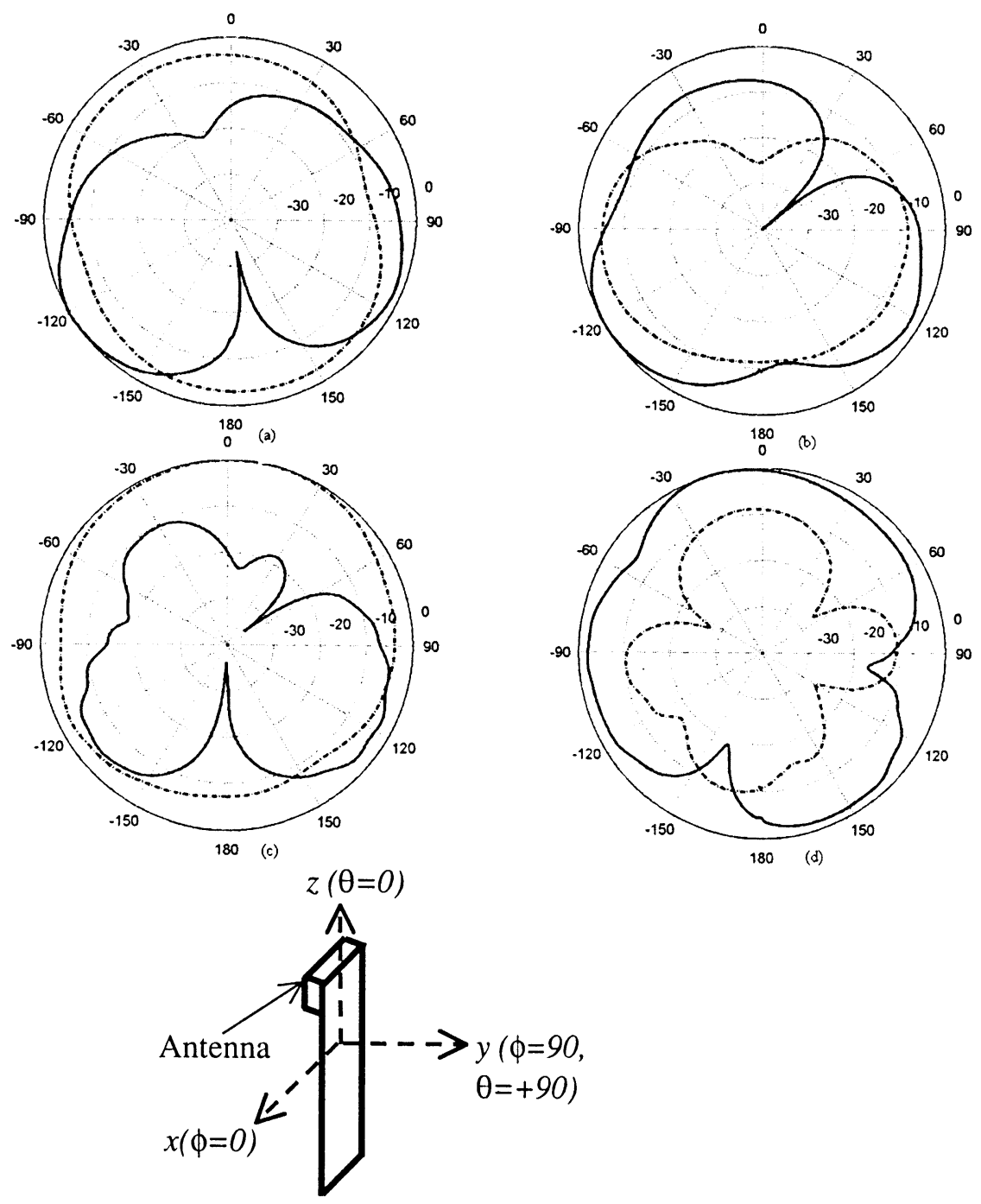

Fig. 10. Measured elevation plane patterns: (a) $y z$ plane (900 MHz), (b) $x z$ plane (900 MHz), (c) $y z$ plane (1900 MHz), and (d) $x z$ plane (1900 MHz). Solid line- vertical component, dashed line- horizontal component; $d=4 \mathrm{~mm}, s=6 \mathrm{~mm}$, and $l=26.5 \mathrm{~mm}$. Other fixed parameters are given in Table $\mathrm{I}$.

TABLE II

Measured Peak Gain Data for the Proposed Antenna (Free Space) at 900 And 1900 MHz. For Pattern Characteristics and Beam Peak LOCATIONS For $y z$ AND $x z$ Planes (SEe Fig. 10)

\begin{tabular}{c|c|c}
\hline Frequency $(\mathbf{M H z})$ & Peak gain (dBi), yz-plane & Peak gain (dBi), xz-plane \\
\hline 900 & -0.5 at $\theta=-120^{\circ}$ & 0.0 at $\theta=-120^{\circ}$ \\
\hline 1900 & 2.3 at $\theta=0^{\circ}$ & 2.5 at $\theta=-16^{\circ}$ \\
\hline
\end{tabular}

Antenna gain and patterns were measured with reference to two standard gain antennas (gain of which were known from the manufacturer's data sheet). For the $900 \mathrm{MHz}$ band a log-periodic dipole antenna and for the $1900 \mathrm{MHz}$ band a rectangular horn antenna was used. Measured gain for the two principal plane patterns of the proposed antenna are listed in Table II. It is apparent that the free-space peak gain at $900 \mathrm{MHz}$ is between 0 and $-0.5 \mathrm{dBi}$ and is directed toward $\theta=-120^{\circ}$ [for antenna orientation and pattern shape see Fig. 10(a) and (b)]. This is rather typical for cellular phone antennas, especially at around $900 \mathrm{MHz}$. The peak of the beam is generally directed toward the lower hemisphere since the PCB which itself radiates, is much larger than the actual antenna and, thus, dominates the pattern [9]. The gain at $1900 \mathrm{MHz}$ is between 2.3 and $2.5 \mathrm{dBi}$ and is directed toward $\theta=0^{\circ}$ [for antenna orientation and pattern shape see Fig. 10(c) and (d)]. This is also expected since the antenna is more directional in the high band. Also since the antenna is positioned asymmetrically to the top end of the PCB the peak of the beam is along $\theta=0^{\circ}$.

Measured normalized radiation pattern data for the proposed antenna are shown in Figs. 9 and 10. The azimuth ( $x y$ plane $\theta=90^{\circ}$ ) patterns at 900 and $1900 \mathrm{MHz}$ are shown in Fig. 9(a) and (b). At $900 \mathrm{MHz}$ the vertical component of field is the dominant one [Fig. 9(a)] and its variation is nearly uniform. The front to back ratio is about $3 \mathrm{~dB}$. At $1900 \mathrm{MHz}$ [Fig. 9(b)] the vertical component of field is fairly directional, front to 
back ratio is about $8 \mathrm{~dB}$. Even though the vertical component is not uniform fairly good angular coverage can still be obtained when both vertical and horizontal components are combined. The only angular region where coverage is between -8 to $-10 \mathrm{~dB}$ is limited between the angular region of 60 to $120^{\circ}$. This angular region is going to be blocked by the operator head anyway. Thus, the directionality in the high band can be considered as an advantage since less energy is being deposited in the operator head. Another thing to note is the significance of total field rather than just one component. In a mobile environment polarization purity is virtually absent. Thus, when both components exist and are comparable they need to be combined to get the total field.

Measured elevation plane gain patterns for the proposed antenna are shown in Fig. 10. The $y z$ plane pattern at $900 \mathrm{MHz}$ in Fig. 10(a) has both the vertical and horizontal components. Clearly the pattern for the vertical component resembles the pattern of a stub antenna on a box [9]. The horizontal component appears to be more uniform. The $x z$ plane pattern at $900 \mathrm{MHz}$ is shown in Fig. 10(b). The dominant component here is the vertical field component. Once again the pattern looks similar to a stub antenna pattern.

Patterns at $1900 \mathrm{MHz}$ are shown in Fig. 10(c) and (d). In the $y z$ plane $\left(\phi=90^{\circ}\right)$ the horizontal field component is the dominant one with nearly uniform variation between $0^{\circ}$ to $90^{\circ}$ and $-90^{\circ}$ to $0^{\circ}$ (between 0 to $-4 \mathrm{~dB}$ ). The vertical component shows a butterfly-type pattern mainly because the PCB and antenna combination is longer than a wavelength at this frequency. When both fields are combined the total field should still be similar to the horizontal component shown in Fig. 10(c). The $x z$ plane pattern [Fig. 10(d)] shows that the vertical component of field is the dominant one and, thus, the total field pattern should resemble that.

\section{CONCLUSION}

The analysis and design of a multiband internal antenna is presented. The antenna consists of a driven meander-line element and two coupled parasitic elements. It has been demonstrated that the antenna can operate either in the AMPS 800/GSM 900/GSM 1900 or the AMPS 800/GSM 900/GSM $1800 \mathrm{MHz}$ bands within a VSWR of 2.5:1. The principle described here can be exploited to design antennas for other triple or multiband operations. The study presented here does not contain the effect of the user's head and hand on the antenna impedance and pattern characteristics. Since the human brain is a lossy dielectric, electromagnetic absorption will reduce the antenna gain in the direction of the head. The amount of absorption and its effect on antenna pattern depend on multiple factors, such as, operating frequency, antenna design, device size, proximity of antenna from the user's head, and so on. In terms of the antenna input characteristic the user's brain being a dielectric material will reduce the resonant frequency of the antenna, which can be mitigated by making the antenna electrically shorter accordingly. We plan to address the effect of the user's head and hand on the the proposed antenna through future publication.

\section{REFERENCES}

[1] C. R. Rowell and R. D. Murch, "A capacitively loaded PIFA for compact mobile telephone handsets," IEEE Trans. Antennas Propagat., vol. 45, pp. 837-842, May 1997.

[2] M.-S. Tong, M. Yang, Y. Chen, and R. Mittra, "Finite-difference time-domain analysis of a stacked dual-frequency microstrip planar inverted-F antenna for mobile telephone handsets," IEEE Trans. Antennas Propagat., vol. 49, pp. 367-376, Mar. 2001.

[3] G. K. H. Lui and R. D. Murch, "Compact dual-frequency PIFA designs using LC resonators," IEEE Trans. Antennas Propagat., vol. 49, pp. 1016-1019, July 2001.

[4] M. T. Huynh and W. L. Stutzman, "Ground plane effects on PIFA antennas," USNC/URSI Radio Science. Meeting Digest, p. 223, 2000.

[5] IE3D, Zeland Software. IE3D User's Manual Release 8, Zeland Software Incorporated.

[6] M. Ali, S. S. Stuchly, and K. Caputa, "A wide-band dual meandersleeve antenna," J. Electromagn. Waves Applicat., vol. 10, no. 9, pp. 1223-1236, 1996.

[7] M. Ali, M. Okomewski, M. A. Stuchly, and S. S. Stuchly, "Dual-frequency strip-sleeve monopole for laptop computers," IEEE Trans. Antennas Propagat., vol. 47, pp. 317-323, Feb. 1999.

[8] M. Ali, "Dual-Band Antenna Having Mirror Image Meandering Segments and Wireless Communicators Incorporating Same," U.S. patent 6184836 , Feb. 6, 2001.

[9] Mobile Antenna Systems Handbook, K. Fujimoto and J. R. James, Eds., Artech House, Norwood, MA, 1994.

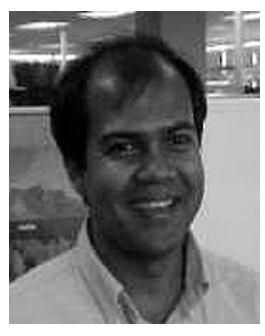

Mohammod Ali (M'00) received the B.Sc. degree in electrical and electronic engineering from the Bangladesh University of Engineering and Technology, Dhaka, in 1987 and the M.A.Sc. and $\mathrm{Ph} . \mathrm{D}$. degrees in electrical engineering from the University of Victoria, BC, Canada, in 1994 and 1997, respectively.

From 1988 to 1992, he worked at the Bangladesh Institute of Technology, Chittagong. From January 1998 to August 2001, he was with Ericsson Incorporated, Research Triangle Park, NC, first as a Staff Engineer and then as a Senior Staff Engineer. Since August 2001, he has been an Assistant Professor with the Department of Electrical Engineering, University of South Carolina, Columbia. He is the author or coauthor of more than 35 journal and conference papers, has four patents in his name, and several patent applications that are pending. His research interests are in the general areas of antennas and RF/microwave engineering. This includes analysis and design of microwave circuits and antennas for wireless communications and defense applications, numerical electromagnetics, and microwave measurements.

Dr. Ali received the National Science Foundation (NSF) Faculty Career Award in 2003.

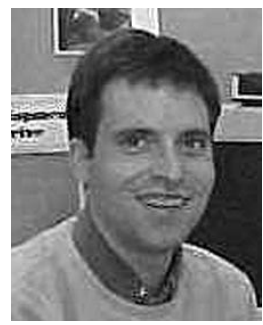

Gerard James Hayes (S'84-M'89) received the B.S. degree in electrical engineering and the M.S. degree in applied mathematics from North Carolina State University, Raleigh, in 1987 and 1993, respectively, and the M.S. degree in electrical engineering from The Ohio State University, Columbus, in 1989.

Previously, he worked as an Antenna Engineer for Lockheed Missiles and Space Corporation, Sunnyvale, CA. Since 1993 he has worked in the Cellular Phone Division, Ericsson, Research Triangle Park, NC. Currently, he is the Technical Manager for the Antenna Development Group for Sony Ericsson Mobile Communications Incorporated, Research Triangle Park. He has multiple U.S. patents in antenna and mobile phone technology, and several patents pending. His research interests include multiband, internal cell phone antennas, 3-D measurement facilities, SAR, and numerical simulation tools. 


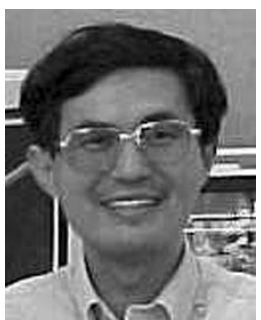

Huan-Sheng Hwang (S'93-M'96) received the M.S.E.E. and Ph.D. degrees from North Carolina State University, Raleigh, in 1993 and 1998, respectively.

In 1998, he joined Raychem Corporation, Fuquay-Varina, NC, where he was an RF Engineer for research and development in RF and microwave circuits. Currently, he is with Sony Ericsson Mobile Communications Incorporated, Research Triangle Park, NC, as a Senior Staff Engineer for antenna design and research for cellular phones and wireless

applications.

Dr. Hwang won the prestigious Bronze Medallion for Outstanding Scientific Achievement presented at the 20th Army Science Conference in 1996. He is also the coauthor of the chapter, Dielectric Slab Combiners, in the book Active and Quasi-Optical Arrays for Solid-State Power Combing. His research interests include quasioptical and spatial power combining systems, antenna designs, electromagnetics, and wave propagations, RF and microwave/millimeter-wave components and circuits.

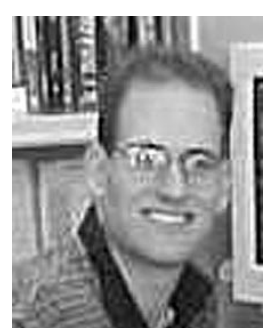

Robert A. Sadler (M'96) received the B.S. degree in electrical engineering from the Virginia Polytechnic Institute and State University, Blacksburg, VA, in 1995 and the M.S.E.E. from North Carolina State University, Raleigh, in 2000

Since 1995, he has worked in the Cellular Phone Division, Ericsson, Research Triangle Park, NC. Currently, he is a Senior Staff Engineer for the Antenna Development Group, Sony Ericsson Mobile Communications Incorporated. He has 10 U.S patents in cell phone antenna technology, and several patents pending. His research interests include internal cell phone antennas, 3-D measurement facilities, SAR, and antenna system simulations using IE3D and SEMCAD. 